\title{
Erratum: Photoionization of hydrogen atoms by coherent intense high-frequency short laser pulses: Direct propagation of electron wave packets on large spatial grids [Phys. Rev. A 88, 023422 (2013)]
}

\author{
Philipp V. Demekhin, David Hochstuhl, and Lorenz S. Cederbaum \\ (Received 5 April 2017; published 17 April 2017)
}

DOI: 10.1103/PhysRevA.95.049903

A short while ago, we found an unfortunate error in the computer code we used in our work to propagate the electronic wave packets. The origin of the error is trivial: It is caused by an almost invisible difference in writing the number " 1 " and the small letter "l". The latter served as an input parameter in the code to assign the maximal value $\ell_{\max }$ in the partial-wave expansion of the electronic wave function in Eq. (3) of our paper. Because of this error, this expansion was restricted in the computations to $\ell_{\max }=1$ (one), i.e., only the partial waves $\ell=0$ and $\ell=1$ were involved in the computations, although a much larger value was used as an input parameter. As a consequence, the electron spectra shown in Figs. 2-4 of our paper are not converged and correspond to angular momenta $\ell=0$ and $\ell=1$ only.

Very recently, Baghery et al. [1] studied the same problem of $1 s$ photoionization of hydrogen atom by coherent intense high-frequency short laser pulses, employing different methods and codes for the numerical solution of the time-dependent Schrödinger equation. The correctly converged $1 s$ photoelectron spectra of hydrogen can be found in Ref. [1]. The results do not exhibit dynamic interference effects. However, these authors clearly demonstrate that dynamic interference does exist for the same system, i.e., a hydrogen atom, but for a different initial state, namely, $2 p$ instead of $1 s$.

We would like to stress that the error in the code does not affect our calculations of dynamic interference in other situations since different computer codes have been used there. These are as follows: (i) resonant Auger decay spectra of Ne and Xe atoms induced by free-electron laser pulses [2-4]; (ii) sequential two-photon ionization of $\mathrm{H}$ atoms by intense resonant laser pulses [4]; (iii) direct ionization of the He atom by intense high-frequency laser pulses where the effect is produced exclusively by collective two-electron dynamics [5]. The phenomenon of dynamic interference is a very general and fundamental effect which emerges in many systems interacting with coherent short laser pulses, and as such, it is not affected by the discussed above error.

[1] M. Baghery, U. Saalmann, and J.-M. Rost, Phys. Rev. Lett. 118, 143202 (2017).

[2] P. V. Demekhin and L. S. Cederbaum, Phys. Rev. A 83, 023422 (2011).

[3] A. D. Müller and P. V. Demekhin, J. Phys. B: At. Mol. Opt. Phys. 48, 075602 (2015).

[4] P. V. Demekhin and L. S. Cederbaum, Phys. Rev. A 86, 063412 (2012).

[5] A. N. Artemyev, A. D. Müller, D. Hochstuhl, L. S. Cederbaum, and P. V. Demekhin, Phys. Rev. A 93, 043418 (2016). 